\title{
Influence of thermal shear localization on elastohydrodynamic contacts
}

\author{
R. Guilbault \\ Department of Mechanical Engineering, École de technologie supérieure, \\ Montreal, Quebec, Canada
}

\begin{abstract}
The temperature in elastohydrodynamic contacts determines the resistance of the lubricant film. Therefore, an efficient assessment of the scuffing risks requires accurate contact temperature prediction. The work presented in this paper makes use of a simple temperature model to investigate the influence of thermal shear localization on the temperature distribution in lubricant films. The model incorporates a multilayer lubricant film representation for the heat equation solution. The paper includes an original heat repartition factor expression and a simple formula for handling intermediate values of the Peclet number. The study also integrates an inlet temperature rise equation. The temperature predictions for sliding/rolling conditions are compared to published numerical results neglecting the shear localization influence. The results agree remarkably well; for the evaluations presenting the higher absolute difference, the correspondence remained over $94 \%$ and $95 \%$ for the maximum and mean temperatures, respectively. For the tested conditions, subsequent inclusion of the thermal shear localization contribution reveals that the shear localization augments the mean film temperature, while, depending on the pressure, the shearing zone occupies between 50 to $70 \%$ of the film thickness.
\end{abstract}

Keywords: elastohydrodynamic, temperature, inlet temperature, heat repartition.

\section{Introduction and literature survey}

The general EHL process has been studied in numerous papers over the years. Some of the published papers are surveyed next. Crook experimentally studied the temperature distribution influence in the oil film between rollers [1,2], and, in agreement with Archard's assumption [3], concluded that the convection 
mechanism presents a negligible contribution. Later, Smith [4, 5] suggested that, exposed to very high pressures, the lubricant could exhibit a solid-like behavior, and over a critical shear stress would shear at a constant stress.

Smith's shear localization hypothesis was subsequently experimentally evidenced by Bair and his collaborators [6-8]. Thermal shear localization appears to be easily identified [9]. Rashid and Sereig [10, 11] published a model for estimation of heat partition in layered contacts. They assumed a liquid portion in the film in the range of 10 to $40 \%$ of the film thickness.

Numerical investigations have addressed the thermal EHL problem [12-14]. Sadeghi and Sui [12] presented a complete numerical solution. Their results were later reproduced with good correspondence by Lee and Hsu [13] and Hsu and Lee [14].

Greenwood and Kauzlarich [15] examined the influence of the inlet shear heating on the film thickness under pure rolling conditions. Later, Murch and Wilson [16] derived a thermal correction factor for pure rolling conditions. This factor was afterward extended to account for sliding conditions [17] and modified by Gupta et al. [18] to integrate the load influence.

The model included in this paper concentrates on the energy equation. Therefore, the thermal film thicknesses are calculated with the isothermal Pan and Hamrock formula (eq. (1)) for the line contact [19], combined with the Gupta thermal correction factor [18] (eq. (2)). The pressure and temperature effects on the viscosity are integrated with the Roeland expression (eq. (3)). The pressure distribution and the contact width are assumed to correspond to the Hertz theory. The investigation aims to illustrate the influence of thermal shear localization on the temperature distribution in lubricant films.

$$
h_{\mathrm{c}}=R\left(2.922 W_{\mathrm{d}}^{-0.166} U_{\mathrm{d}}^{0.692} G_{\mathrm{d}}^{0.47}\right)
$$

where $W_{\mathrm{d}}=\frac{w}{2 E^{*} R}$ is the dimensionless load; $w$ is the load per unit width $[N / m]$; $R\left(\frac{1}{R}=\frac{1}{R_{1}}+\frac{1}{R_{2}}\right)$ is the equivalent radius of the rollers $[m] ; U_{\mathrm{d}}=\frac{\mu_{0} \bar{u}}{2 E^{*} R}$ is the dimensionless speed; $G_{\mathrm{d}}=2 \alpha E *$ is the dimensionless material parameter; $E^{*}=\left(\frac{1-v_{1}^{2}}{E_{1}}+\frac{1-v_{2}^{2}}{E_{2}}\right)^{-1} ; \mu_{0}$ is the dynamic viscosity at atmospheric pressure $[\mathrm{Pa} \cdot s]$; $\alpha$ is the pressure-viscosity coefficient $\left[\mathrm{m}^{2} / N\right]$;

$$
C=\frac{1-13.2\left(\frac{p_{0}}{2 E^{*}}\right) L^{* 0.42}}{1+0.213\left(1+2.23 S_{r}^{0.83}\right) L^{* 0.64}}
$$

where $S_{r}=\frac{\bar{u}}{u_{s}}$ is the slip ratio; $\bar{u}=\frac{u_{1}+u_{2}}{2}$ is the rolling speed; $u_{s}=u_{1}-u_{2}$ is the sliding speed; $u_{1}$ and $u_{2}$ are the tangential speeds and $L^{*}=-\left(\frac{\partial \mu_{1}}{\partial T}\right) \frac{\bar{u}^{2}}{k}$. 


$$
\mu_{1}=\mu_{0} \exp \left\{\left(\ln \mu_{0}+9.67\right)\left[\left(\frac{T-138}{T_{0}-138}\right)^{-S_{0}}\left(1+5.097 \times 10^{-9} p\right)^{z^{*}}-1\right]\right\}
$$

where $p$ is the pressure $[\mathrm{Pa}], T_{0}$ and $T$ are the temperatures $[\mathrm{K}]$ associated with $\mu_{0}$ and $\mu_{1}[\mathrm{~Pa} s]$, respectively. $S_{0}$ is a dimensionless constant representing the slope of the viscosity-temperature relationship and $z^{*}$ is the dimensionless viscositypressure index.

The precision of the temperature model is illustrated through a comparison of the calculated temperatures to the temperature values published by Sadeghi and Sui [12] and Lee and Hsu [13] and Hsu and Lee [14].

\section{Temperature model}

Under the heat generation influence, the shear layer of the lubricant presents a liquid behavior.

\subsection{Contact zone}

A multilayer lubricant film representation similar to the Rashid and Sereig $[10,11]$ model is considered for the heat equation (eq. (4)) treatment. The following hypotheses are also included in the developments: only the conduction cooling across the film thickness is considered [3]; the heat generation is concentrated in the shearing layer; The shearing layer presents a liquid behavior.

Figure 1 illustrates the variables. Both global $(x, y, z)$ and local $\left(x_{1}, y_{l}, z_{l}\right)$ coordinate systems are located at the initial line of contact position. However, the former is situated at the midposition in the film thickness, while the latter is attached to the midposition of the shearing layer.

$$
K \frac{\partial^{2} T}{\partial z^{2}}+H=0
$$

where $H$ is given by eq. (5).

$$
H=\mu \dot{\gamma}^{2}
$$

where $T$ is the temperature $[K], K$ is the thermal conductivity $[\mathrm{W} /(\mathrm{mK})], H$ is the heat source or viscous dissipation $\left[\mathrm{W} / \mathrm{m}^{3}\right], \mu$ the viscosity $[\mathrm{Pa} \mathrm{s}]$.

The factors $f$ and $g$ define the relative shearing layer thickness and position, respectively. $T_{B i}, T_{S i}$ and $T_{f i}$ are the bulk, the surface and the solid/shearing zone interface temperatures, respectively.

The following expressions establish the relation between the variables:

$$
\begin{gathered}
z_{1}=z-g \frac{h}{2} \\
\frac{-h}{2}(1+g) \leq z_{1} \leq \frac{h}{2}(1-g)
\end{gathered}
$$


6 Tribology and Design II

$$
|g| \leq|1-f|
$$

If $\beta$ represents the heat proportion flowing to solid 1, eq. (4) can be solved considering the heat partition. In zones $l_{1}$ and $l_{2} H=0$. Consequently, the temperature $T$ presents a linear distribution $\left(\frac{\partial^{2} T}{\partial z^{2}}=0\right)$. Considering the Fourier's law (eq. (7)), and integrating, eq. 8 gives the temperature distribution.

$$
\begin{gathered}
q=-K \frac{\partial T}{\partial z_{1}} \\
T=-\frac{q z_{1}}{K}+c
\end{gathered}
$$

where $c$ is an integration constant. Since $H$ and $q$ (the heat flux $\left[\mathrm{W} / \mathrm{m}^{2}\right]$ ) are related by the first thermodynamic law (eq. (9)), replacing $q$ and considering that ( $T\left(z_{1}=f \frac{h}{2}\right)=T_{f 1}$ ) lead to eq. (10). Equation (10) may also be rewritten in terms of $T_{S I}$ (eq. (11)). Equations (10) and (11) are valid in the solid-like zone $l_{1}$ $\left(z_{1} \geq f \frac{h}{2}\right)$.

$$
\begin{gathered}
\frac{\partial q}{\partial z}+H=0 \\
T\left(z_{1}\right)=\beta \frac{H h}{K}\left(f \frac{h}{2}-z_{1}\right)+T_{f 1} \\
T\left(z_{1}\right)=\beta \frac{H h}{K}\left(\frac{h}{2}(1-g)-z_{1}\right)+T_{S 1}
\end{gathered}
$$

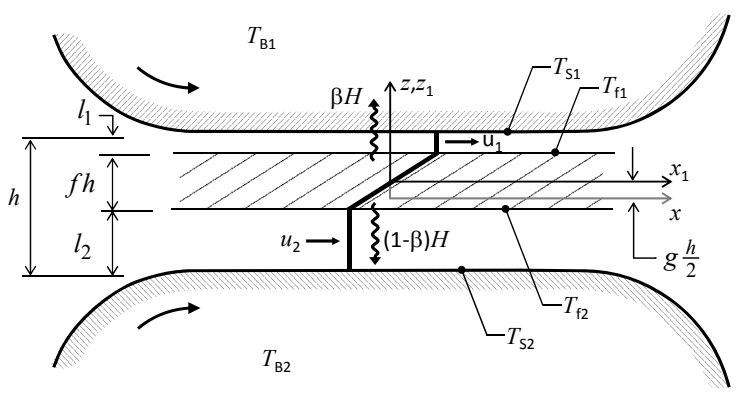

Figure 1: Multilayer film representation. 
Equation (8) also serves to define the temperature distribution in zone $l_{2}$. Thus, considering that $T\left(z_{1}=-f \frac{h}{2}\right)=T_{f 2}$ and the remaining heat portion $(1-\beta) H$ flowing to solid 2, eqs. (12) and (13) give the temperature distribution as a function of the solid-shearing zone interface temperature $\left(T_{f 2}\right)$, and in terms of $T_{S 2}$, respectively. Equations (12) and (13) are valid in the solid-like zone $l_{2}$ $\left(z_{1} \leq-f \frac{h}{2}\right.$ ). In eqs. (10) and (11) or (12) and (13), $K$ has to be evaluated in the considered zone.

$$
\begin{gathered}
T\left(z_{1}\right)=(1-\beta) \frac{H h}{K}\left(f \frac{h}{2}-\left|z_{1}\right|\right)+T_{f 2} \\
\left.T\left(z_{1}\right)=(1-\beta)\right) \frac{H h}{K}\left(\frac{h}{2}(1+g)-\left|z_{1}\right|\right)+T_{S 2}
\end{gathered}
$$

It is assumed that the heat source is located in the shearing layer ( $f h)$. Therefore, successive integrations of eq. (4) considering the boundary conditions $T\left(z_{1}=f \frac{h}{2}\right)=T_{f 1}$ and $T\left(z_{1}=-f \frac{h}{2}\right)=T_{f 2}$ lead to the temperature distribution in eq. (14). Replacing $T_{f 1}$ and $T_{f 2}$ in eq. (14) gives the formulation presented in eq. (15). The average temperature in in the shearing zone is calculated from eq. (16). The complete temperature distribution is given by eqs. (11), (13) and (15).

$$
\begin{gathered}
T\left(z_{1}\right)=\frac{H}{2 K}\left(\left(f \frac{h}{2}\right)^{2}-\left(z_{1}\right)^{2}\right)+\left(\frac{T_{f 1}-T_{f 2}}{f h}\right) z_{1}+\left(\frac{T_{f 1}+T_{f 2}}{2}\right) \\
T\left(z_{1}\right)=\frac{H}{2 K}\left(\left(f \frac{h}{2}\right)^{2}-\left(z_{1}\right)^{2}\right)+\left(\frac{T_{S 1}-T_{S 2}+\frac{H h^{2}}{2 K}((2 \beta-1)(1-f)-g)}{f h}\right) z_{1}+\left(\frac{T_{s 1}+T_{S 2}}{2}\right)+\frac{H h^{2}}{4 K}((1-2 \beta) g+1-f) \\
T_{\text {ave }}=\frac{1}{f h} \int_{-f \frac{h}{2}}^{f \frac{h}{2}} T\left(z_{1}\right) d z_{1}=\frac{H}{4 K}\left(\frac{(f h)^{2}}{3}+h^{2}((1-2 \beta) g+1-f)\right)+\left(\frac{T_{S 1}+T_{S 2}}{2}\right)
\end{gathered}
$$

\subsection{Heat repartition}

Heat propagation from the source to the solid bodies is described by the thermal circuit shown in Fig. 2, where the $L$ variables are the thicknesses given in eqs. (17) and the $R_{i j}$ are thermal resistances defined by eqs. (19) to (20). $R_{i l}$ and $R_{i 2}$ are the resistances of the shearing zone and solid-like portion of the film, respectively, while $R_{i 3}$ represents the temperature gradient between the surfaces and bulks. 
8 Tribology and Design II

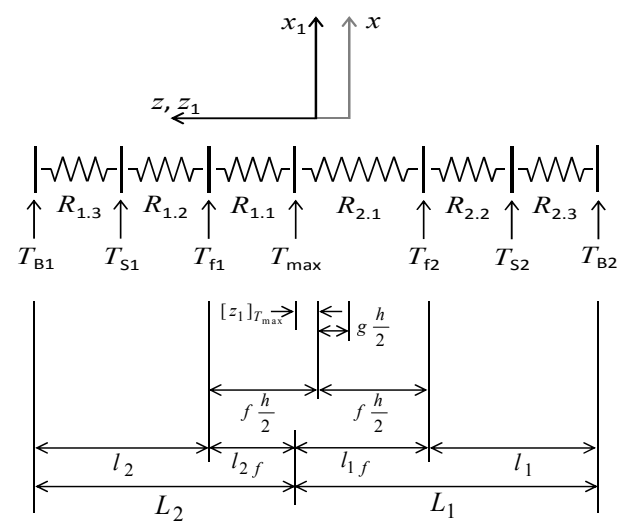

Figure 2: $\quad$ Oil film thermal circuit.

$$
\begin{array}{lll}
l_{1 f}=f \frac{h}{2}-\left[z_{1}\right]_{T_{\max }} & \text { (17.a) } & l_{2 f}=f \frac{h}{2}+\left[z_{1}\right]_{T_{\max }} \\
l_{1}=L_{1}-l_{1 f}=\frac{h}{2}(1-f-g) & (17 . \mathrm{c}) & l_{2}=L_{2}-l_{2 f}=\frac{h}{2}(1-f+g) \\
L_{1}=\frac{h}{2}(1-g)-\left[z_{1}\right]_{T_{\max }} & (17 . \mathrm{e}) & L_{2}=\frac{h}{2}(1+g)+\left[z_{1}\right]_{T_{\max }} \\
R_{1.1}=\frac{l_{1 f}}{K_{\text {lub-f }}} & \text { (18.a) } & R_{2.1}=\frac{l_{2 f}}{K_{\text {lub- }-f}} \\
R_{1.2}=\frac{l_{1}}{K_{\text {lub }-S 1}} & \text { (19.a) } & R_{2.2}=\frac{l_{2}}{K_{\text {lub-S2 }}} \\
R_{1.3}=\frac{\Phi_{1} \chi_{1}}{K_{1} u_{1}} & \text { (20.a) } & R_{2.3}=\frac{\Phi_{2} \chi_{2}}{K_{2} u_{2}}
\end{array}
$$

where $\chi\left[\mathrm{m}^{2} / \mathrm{s}\right]$ is the oil thermal diffusivity, $K_{l u b}$ and $K_{l u b-S(1 \text { or 2) }}$ are the lubricant thermal conductivities in the shearing zone (eq. 16) and in the lubricant-solidlike zones. The variables $\Phi_{i}$ are dimensionless temperatures (see next section).

Considering that $R_{1}=\sum_{i=1}^{3} R_{1 . i}$ and $R_{2}=\sum_{i=1}^{3} R_{2 . i}$, the heat partition factor is calculated from eq. (21). 


$$
\beta=\frac{R_{2}}{R_{1}+R_{2}}=\frac{\left(\frac{l_{2 f}}{K_{\mathrm{lub} f}}+\frac{l_{2}}{K_{\mathrm{lub}-S 2}}+\frac{\Phi_{2} \chi_{2}}{K_{2} u_{2}}\right)}{\left(\frac{f h}{K_{\mathrm{lub}}}+\frac{l_{1}}{K_{\mathrm{lub}-S 1}}+\frac{l_{2}}{K_{\mathrm{lub}-S 2}}+\frac{\Phi_{1} \chi_{1}}{K_{1} u_{1}}+\frac{\Phi_{2} \chi_{2}}{K_{2} u_{2}}\right)}
$$

\subsection{Surface temperature}

Jaeger [20] showed that the temperature rise $\left(\theta_{f a}\right)$ provoked by a fast moving rectangular $(2 a \times 2 b)$ heat source (where the Peclet number $P e=\frac{u a}{2 \chi}$ is greater than 5$)$ is given by eq. 22. For a slow-moving rectangular $(2 a \times 2 b)$ heat source $(P e<<1)$, the mean temperature rise $\left(\theta_{s l}\right)$ was established by Loewen and Shaw (as reported by Bushan [21]).

$$
\begin{gathered}
\theta_{f a}=T_{S}-T_{B}=\frac{4}{3 \sqrt{\pi P e}} \frac{a}{K} q \\
\theta_{s l}=T_{S}-T_{B}=\bar{A} \frac{a}{K} q
\end{gathered}
$$

where $\bar{A}$ is given by eq. (24).

$$
\bar{A}=0.64\left\{\frac{b}{a} \sinh ^{-1}\left(\frac{a}{b}\right)+\sinh ^{-1}\left(\frac{b}{a}\right)+0.33\left(\frac{b}{a}\right)^{2}+0.33 \frac{a}{b}-0.33\left(\frac{a}{b}+\frac{b}{a}\right) \sqrt{1+\left(\frac{b}{a}\right)^{2}}\right\}
$$

For the moderate speed range $(0.1 \leq P e \leq 10)$, the temperature rise has to be interpolated. Equation (25) presents the interpolation form suggested by Churchill and Usagi [22]. Greenwood [23] demonstrated that $e=2$ offered excellent correspondence with Jaeger's evaluations.

$$
\frac{1}{\Phi^{e}}=\frac{1}{\Phi_{s l}^{e}}+\frac{1}{\Phi_{f a}^{e}}
$$

If, following Greenwood's suggestion [23], $P e$ is replaced by $J=\frac{u a}{\chi}$, the combination of eqs. (23) and (24) accordingly to eq. (25) with $e=2$ gives the dimensionless temperature evaluation for any $P e$ number (eq. (26)).

$$
\Phi=B J\left\{\left(\frac{B}{\bar{A}}\right)^{2}+J\right\}^{-\frac{1}{2}}
$$

where $B=\frac{4}{3} \sqrt{\frac{2}{\pi}}$. 


\subsection{Inlet temperature}

For rolling/sliding conditions, successive integrations of the heat equation (eqs. (4) and (5)), considering the boundary conditions as $T=T_{S}$ at $z= \pm h / 2$ and $\dot{\gamma}$ given by eq. (27) for the global coordinate system of Fig. 1 leads to the inlet temperature distribution (eq. (28)).

$$
\begin{aligned}
& \dot{\gamma}=\frac{\partial u}{\partial z}=\frac{z}{\mu} \frac{\partial p}{\partial x}+\frac{u_{1}-u_{2}}{h} \\
&=\Gamma_{1} z+\Gamma_{2} \\
& T-T_{S}=\Delta T=-\frac{\mu}{K}\left\{\frac{\Gamma_{1}}{12}\left(z^{4}-\left(\frac{h}{2}\right)^{4}\right)+\frac{\Gamma_{1} \Gamma_{2}}{6}\left(z^{3}-\left(\frac{h}{2}\right)^{2} z\right)+\frac{\Gamma_{2}^{2}}{2}\left(z^{2}-\left(\frac{h}{2}\right)^{2}\right)\right\}
\end{aligned}
$$

where $h$ in the inlet may be assumed to vary from $h_{\infty}$ far from the contact zone to $h_{\mathrm{c}}$ in the contact zone $(x=-a)$. Moreover, since secondary inlet flows mix the lubricant, the averaged value of $\Delta T$ offers a reasonable evaluation of the inlet lubricant temperature. If eq. (28) is integrated with respect to $z$, the average temperature increase across the film thickness is given by eq. (29).

$$
\frac{1}{h} \int_{-\frac{h}{2}}^{\frac{h}{2}} \Delta T d z=\frac{\mu h^{2}}{48 K}\left\{\frac{h^{2}}{5} \Gamma_{1}^{2}+5 \Gamma_{2}^{2}\right\}
$$

The average inlet temperature increase is finally obtained by the integration of eq. (29) along the $x$ direction. Following the Greenwood and Kauzlarich [15] procedure, the Reynolds equation (eq. (30)) and the substitution eq. (31) are replaced into eq. (29). The Barrus eq. (32) is also incorporated into eq. (29). To ease the manipulations, Greenwood and Kauzlarich [15] proposed the approximation given in eq. (33) and considered the Grubin approximation (infinite pressure at $x=-a$ ). The Poiseuille term $\left(\Gamma_{1}\right)$ reduces to zero at $\kappa=0$. Therefore, since Greenwood only accounted for $\Gamma_{1}$, the combination with the Grubin assumption integrated between $\kappa=0$ and $\kappa=\pi / 2$ lead to a finite value. In contrast, $h^{2} \Gamma_{2}^{2}$ is independent of $\kappa$. The integral of $h^{2} \Gamma_{2}^{2}$ thus reaches an infinite value at $\kappa=0$. In reality, the infinite pressure hypothesis was introduced with negligible effects for conditions where $\alpha p \geq 5$.

$$
\begin{gathered}
\frac{\partial p}{\partial x}=12 \bar{u} \mu\left(\frac{h-h_{c}}{h^{3}}\right) \\
\tan \kappa=\sqrt{\frac{h-h_{c}}{h_{c}}}
\end{gathered}
$$

where at $h(x \rightarrow \infty)=h_{\infty}$ and $\kappa_{1} \simeq \pi / 2$ and at $h(x=-a)=h_{c}$ and $\kappa_{0} \simeq 0$

$$
\mu_{1}=\mu_{0} \exp (\alpha p)
$$




$$
\exp (\alpha p)=\sin ^{-10 / 3}(\kappa)(1-0.328 \cos (\kappa))
$$

In the present study, the boundless integral generated by the infinite pressure simplification is eliminated with a relocation of the inlet-contact zone transition at $\alpha p=5$ instead of $\alpha p=\infty$. Thus, from eq. (33) $\kappa_{0}=0.2$. Therefore, the numerical integration of eq. (29), considering eqns. (30) to (33), gives the average inlet temperature increase under rolling-sliding conditions (eq. (34) below).

$$
\overline{\Delta T}=\frac{1}{\left(\kappa_{1}-\kappa_{0}\right)} \frac{\mu h^{2}}{48 K} \int_{\kappa_{0}=0.2}^{\kappa_{1}=\frac{\pi}{2}} \frac{h^{2}}{5} \Gamma_{1}^{2}+5 \Gamma_{2}^{2} d x=\frac{\mu}{K}\left\{\frac{1}{2.55} \bar{u}^{2}+\frac{51}{48} u_{S}^{2}\right\}
$$

\section{Model solution}

The heat generated in the contact zone $(H)$ is established with eq. (5), while the bulk temperatures $\left(T_{B 1}\right.$ and $\left.T_{B 2}\right)$ are assumed to be known variables. Therefore, $f$ and $g$ represent the unknowns of the model. In this study, it is assumed that the shearing layer appears in the central portion of the film thickness. As a result, $g$ is fixed at 0 . Moreover, postulating that EHL systems always tend to operate with the lowest coefficient of friction ( $\zeta$ ) (eq. (40)) appears to be reasonable. Consequently, the appropriate $f$ value is established when $\zeta$ is at a minimum.

$$
\zeta=\mu \dot{\gamma} / p
$$

\section{Model validation}

A comparison of the maximum and average temperatures calculated in the shearing zone to the numerical values published by Sadeghi and Sui [12] and Lee and Hsu [13] and Hsu and Lee [14] serves to establish the precision of the model for line contact problems. Table 1 gives the geometric definition of the system and the lubricant properties. One dimensionless load $W$ is considered $\left(1.3 \times 10^{-4}\right)$, while four dimensionless speeds $U$ and four slip ratios $S_{r}$ are tested. The reference temperatures [12-14] were obtained from modeling considering no shear localization. Consequently, in the present simulations, $f$ was fixed at 1 . The bulk temperatures of the two disks were set to the oil temperature in the tank. Moreover, the reference studies accounted for the viscosity/pressure-temperature relationship by means of the variant of the Roelands given below (eq. (36)). For this reason, eq. (36) replaces eq. (3) in the simulation. Table 2 compares the calculated maximum and average temperature increases in the shear zone to the reference values.

$$
\mu=\exp \left\{\left[\ln \mu_{0}+9.67\right]\left[-1+\left(1+5.1 \times 10^{-9} p\right)^{z^{*}}\right]+\psi T_{0}(1-T)\right\}
$$


Table 1: $\quad$ Disk system and lubricant properties of ref. [13].

\begin{tabular}{llll}
\hline Disks & \multicolumn{3}{c}{ Lubricant } \\
\hline Equi. radius $R$ & $0.02 \mathrm{~m}$ & Temperature (tank) & $313 \mathrm{~K}$ \\
Poisson coef. $v$ & 0.3 & Viscosity at $313 \mathrm{~K}$ & $0.04 \mathrm{Pas}$ \\
Young mod. $E$ & $200 \mathrm{GPa}$ & Viscosity at $373 \mathrm{~K}$ & $0.003218 \mathrm{~Pa} \mathrm{~s}$ \\
Specific heat $c$ & $460 \mathrm{~J} /(\mathrm{kg} \mathrm{K})$ & Pres-visco. coef. $\alpha$ & $1.59 \mathrm{x}^{-8} \mathrm{~Pa}^{-1}$ \\
Th. conduc. $\chi$ & $47 \mathrm{~W} /(\mathrm{m} \mathrm{K})$ & Tempera.-visco. coef. $\psi$ & $0.042 \mathrm{~K}^{-1}$ \\
Density $\rho$ & $7850 \mathrm{~kg} / \mathrm{m}^{3}$ & Specific heat $c_{\text {lub }}$ & $2000 \mathrm{~J} /(\mathrm{kg} \mathrm{K})$ \\
& & Thermal conductivity $\chi$ & $0.14 \mathrm{~W} /(\mathrm{m} \mathrm{K})$ \\
& & Density $\rho_{\text {lub }}$ at $313 \mathrm{~K}$ & $846 \mathrm{~kg} / \mathrm{m}^{3}$ \\
\hline
\end{tabular}

Table 2: $\quad$ Temperature validation for the dimensionless load $W=1.3 \times 10^{-4}$.

\begin{tabular}{|c|c|c|c|c|c|c|}
\hline \multirow[t]{2}{*}{$\begin{array}{c}\text { Speed } \\
U \\
\end{array}$} & \multirow[t]{2}{*}{$\begin{array}{c}S_{r} \\
{[\%]}\end{array}$} & \multicolumn{3}{|c|}{$\begin{array}{c}\text { Maximum temperature } \\
\text { increase } \\
\end{array}$} & \multicolumn{2}{|c|}{$\begin{array}{l}\text { Maximum average } \\
\text { temperature increase }\end{array}$} \\
\hline & & ref.[12] & ref.[14] & model & ref.[13] & model \\
\hline \multirow[t]{4}{*}{$1.8 \times 10^{-11}$} & 0 & 0.91 & - & 0.43 & - & 0.43 \\
\hline & 10 & 10.64 & - & 14.66 & - & 12.64 \\
\hline & 20 & 31.61 & - & 31.55 & - & 27.17 \\
\hline & 30 & 46.57 & - & 44.55 & - & 38.35 \\
\hline \multirow[t]{4}{*}{$3.6 \times 10^{-11}$} & 0 & 2.76 & - & 1.59 & - & 1.59 \\
\hline & 10 & 26.64 & 27.28 & 28.16 & 21.31 & 22.94 \\
\hline & 20 & 53.46 & 51.17 & 51.02 & 40.0 & 41.38 \\
\hline & 30 & 71.12 & 67.19 & 66.90 & 53.09 & 54.27 \\
\hline \multirow[t]{4}{*}{$5.5 \times 10^{-11}$} & 0 & 6.15 & - & 3.34 & - & 3.34 \\
\hline & 10 & 40.37 & 39.54 & 40.43 & 29.76 & 32.15 \\
\hline & 20 & 69.95 & 67.5 & 66.66 & 50.09 & 52.67 \\
\hline & 30 & 89.10 & 85.5 & 84.11 & 64.46 & 66.45 \\
\hline \multirow[t]{4}{*}{$7.3 \times 10^{-11}$} & 0 & 9.97 & - & 5.27 & - & 5.27 \\
\hline & 10 & 51.33 & 51.18 & 50.26 & 38.77 & 39.59 \\
\hline & 20 & 82.95 & 79.70 & 78.45 & 59.76 & 61.29 \\
\hline & 30 & 102.67 & 99.18 & 96.78 & 73.28 & 75.58 \\
\hline
\end{tabular}


Table 2 evidences the high correspondence between the calculated temperatures and the reference values. Considering the maximum temperature increase, for the sliding cases, a comparison to ref. [12] shows that the maximum difference is $-5.89^{\circ} \mathrm{C}\left(U=7.3 \times 10^{-11}\right.$ and $\left.S_{r}=30 \%\right)$, which corresponds to a relative difference of $-5.74 \%$, while compared to ref. [14], the maximum difference is $-2.40^{\circ} \mathrm{C}$ or $-2.42 \%$. A comparison with ref. [13] gives a maximum difference of $2.58^{\circ} \mathrm{C}\left(U=5.5 \times 10^{-11}\right.$ and $\left.S_{r}=20 \%\right)$, which represent a $4.90 \%$ relative difference. The pure rolling cases demonstrate more visible differences. However, since the reference temperatures are the maximum values in the inlet, whereas the temperatures obtained from eq. 34 are averaged values, the real deviations are of negligible amplitude. The equations developed in this study are therefore considered to offer high precision evaluations of the EHL inlet and film temperatures.

\section{Influence of thermal shear localization}

The simulations are repeated accounting for the minimal friction coefficient condition by adjusting the $f$ amplitude. Figure 3 compares the calculated coefficient of friction to the coefficient published in ref. [12]. The values show a perfect agreement. Figure 4 presents the $f$ values. For the studied conditions, the liquid layer occupies between 50 to $70 \%$ of the film thickness. Figure 5 compares the maximum temperature increases to the evaluations obtained with $f$ fixed at 1 . The empty indicators correspond to the no-shear-localization case, whereas the grey-filled indicators denote the temperatures calculated when considering the shear localization influence. The curves of Fig. 5 (a) indicate that, depending on the speed, the localization may raise the maximum temperature. On the other hand, the shear localization effects generate liquid layers, presenting higher mean temperatures and lower mean viscosities.

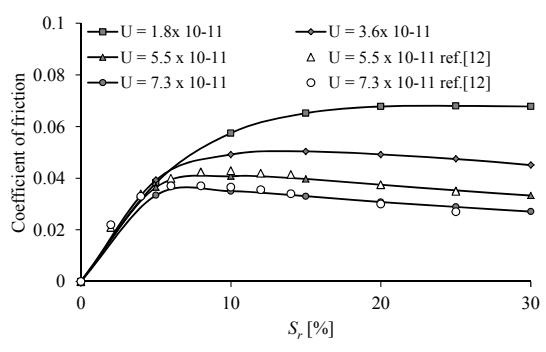

Figure 3: Coefficients of friction.

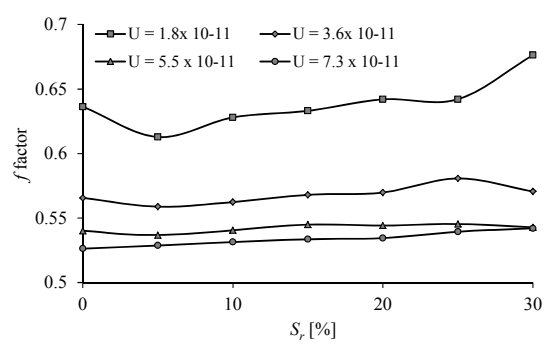

Figure 4: $\quad f$ factor.

The depiction is completed with Figs. 6 (a) and (b) presenting the 2D central $\left(y=0, U=7.3 \times 10^{-11}\right)$ temperature distributions across the film thickness for $S_{r}=30 \%$ when $f$ is fixed at 1 and when $f$ is adjusted, respectively. Figure 6 shows that the thermal shear localization addition draws the temperature distribution towards the slower surface. 


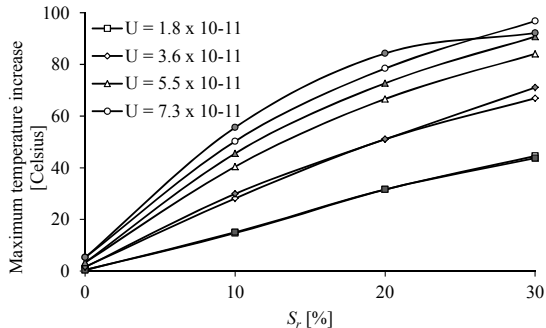

(a) Max. temperature increases

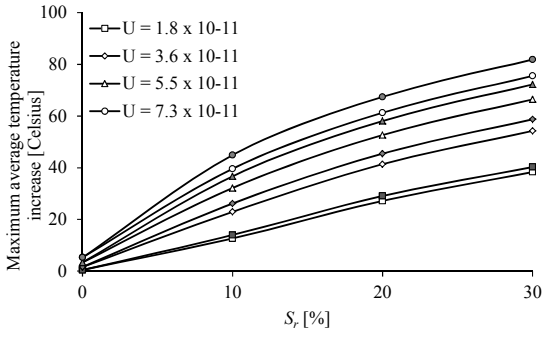

(b) Max. average temperature increases

Figure 5: $\quad$ Temperature increases $\left(W=1.3 \times 10^{-4}\right) f$ set to 1 (empty indicators), $f$ adjusted (grey-filled indicators).

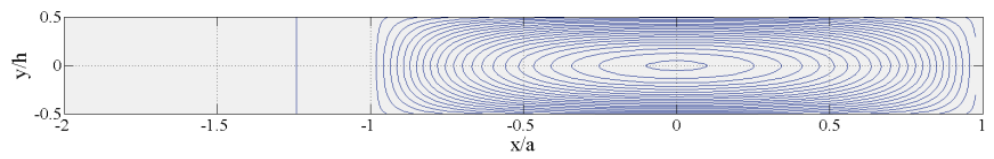

(a) $f$ factor set to 1 (no-shear-localization case)

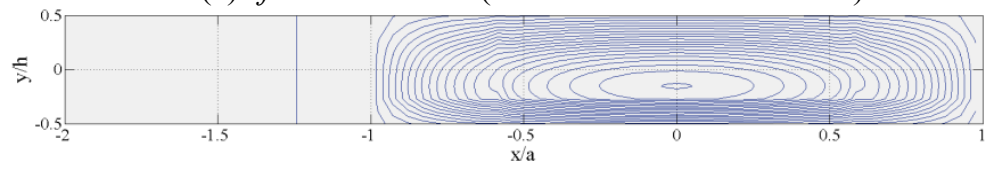

(b) $f$ factor adjusted for shear-localization influence

Figure 6: 2D film temperature distribution $y=0, \quad U=7.3 \times 10^{-11}$ and $S_{r}=30 \%$.

\section{Conclusion}

The work presented in this paper aimed to illustrate the influence of thermal shear localization on the temperature distribution in lubricant film. A simple temperature model serves for the investigation.

The model incorporates a multilayer lubricant film representation for the heat equation solution. The configuration admits liquid and solid phases where the thickness of the liquid shearing layer is controlled by a minimum coefficient of friction condition. The solution only considers the conduction across the film. The temperature model includes an original heat repartition factor expression. A very simple expression is also presented to handle intermediate values of the Peclet number. The rolling/sliding conditions in the inlet provoke a temperature rises. The equation included in this study for inlet temperature rise prediction offered precise temperature evaluations. When neglecting the shear localization 
influence, the contact zone temperature predictions of the model agreed remarkably well with published results. For the evaluations presenting the higher absolute difference, the correspondence remained over $94 \%$ and $95 \%$ for the maximum and mean temperatures, respectively. The subsequent inclusion of the thermal shear localization contribution reveals that shear localization augments the mean film temperature, and therefore, reduces the mean viscosity, and generates lower coefficients of friction. For the studied conditions, the shearing zone occupies between 50 to $70 \%$ of the film thickness.

\section{References}

[1] A.W. Crook, The lubrication of rollers III. A theoretical discussion of friction and the temperatures in the oil film, Philos. Trans. R. Soc. London, Ser. A, 254 (1040), pp. 237-258, 1961.

[2] A.W. Crook, The lubrication of rollers IV. Measurements of friction and effective viscosity, Philos. Trans. R. Soc. London, Ser. A, 255 (1056), pp. 281-312, 1963.

[3] J.F. Archard, The temperature of rubbing surfaces, Wear, 2 (6), pp. 438455, 1959.

[4] F.W. Smith, The Effect of Temperature in Concentrated Contact Lubrication, ASLE Transaction, 5 (1), pp. 142-148, 1962.

[5] F.W. Smith, Rolling contact lubrication - the application of elastohydrodynamic theory, J. Basic Engineering 87 (1), pp. 170-175, 1965.

[6] S. Bair, F. Qureshi, W.O. Winer, Observations of shear localization in liquid lubricants under pressure, Transaction of the ASME Journal of Tribology, 115, pp. 507-513, 1993.

[7] S. Bair, F. Qureshi, M. Khonsari, Adiabatic shear localization in a liquid lubricant under pressure, Transaction of the ASME Journal of Tribology, 116, pp. 705-708, 1994.

[8] S. Bair, C. McCabe, A study of mechanical shear bands in liquids at high pressure, Tribology International, 37, pp. 783-789, 2004.

[9] L. Chang, On the shear bands and shear localizations in elastohydrodynamic lubrication films, Transaction of the ASME Journal of Tribology, 127, pp. 245-247, 2005.

[10] M. Rashid, A. Sereig, Heat repartition and transient temperature distribution in layered concentrated Part I-Theoretical Model, Transaction of the ASME Journal of Tribology, 109, pp. 487-495, 1987.

[11] M. Rashid, A. Sereig, Heat repartition and transient temperature distribution in layered concentrated Part II-Dimensionless relationship and numerical results, Transaction of the ASME Journal of Tribology, 109, pp. 496-501, 1987.

[12] F. Sadeghi, P.C. Sui, Thermal Elastohydrodynamic lubrication of rolling/sliding, Transaction of the ASME Journal of Tribology, 112, pp. 189-195, 1990. 
[13] R.-T. Lee, C.H. Hsu, A fast method for the analysis of thermalelastohydrodynamic lubrication of rolling/sliding line contacts, Wear, 166 (1), pp. 107-117, 1993.

[14] C.H. Hsu, R.-T. Lee, An efficient Algorithm for thermal elastohydrodynamic lubrication under rolling/sliding line contacts, Transaction of the ASME Journal of Tribology, 116, pp. 762-769, 1994.

[15] J.A. Greenwood, J.J. Kauzlarich, Inlet shear heating in elastohydrodynamic lubrication, Transaction of the ASME Journal of Lubrication Technology, 95 (4), pp. 417-426, 1973.

[16] L.E. Murch, W.R.D. Wilson, A thermal elastohydrodynamic inlet zone, Transaction of the ASME Journal of Lubrication Technology, 97 (2), pp. 212-216, 1975.

[17] W.R.D. Wilson, S. Sheu, Effect of inlet shear heating due to sliding on elastohydrodynamic film, Transaction of the ASME Journal of Lubrication Technology, 105 (2), pp. 187-188, 1983.

[18] P.K. Gupta, H.S. Cheng, N.H. Forster, Viscoelastic effects in MIL-L-7808Type lubricant, Part I: Analytical formulation, Tribology transactions, 35 (2), pp. 269-274, 1992.

[19] B.J. Hamrock, S.T. Schmid, B.O. Jacobson, Fundamentals of fluid film lubrication second edition, Marcel Dekker, New York, United States, Chap. 18 and 19, 2004.

[20] J.C. Jaeger, Moving sources of heat and the temperature at sliding contacts, J. and Proc. Roy. Soc. New South Wales, 76, pp. 203-224, 1942.

[21] B. Bushan, Principles and applications of tribology, John Wiley and Sons, New York, United States, Chap. 7, 1999.

[22] S. W Churchill, R. Usagi, A general expression for the correlation of rates of transfer and other phenomena, AICHE Journal, 18 (6), pp.1121-1128, 1972.

[23] J.A. Greenwood, An interpolation formula for flash temperatures, Wear, 150 (1-2), pp.153-158, 1991. 